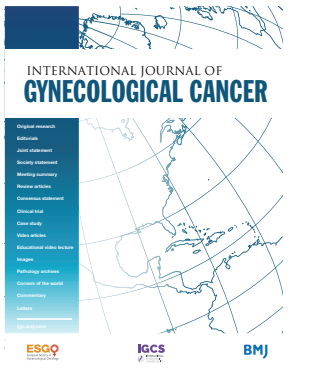

'Department of Obstetrics and Gynecology, The University of Utah, Salt Lake City, Utah, USA ${ }^{2}$ Department of Radiation Oncology, Huntsman Cancer Institute, Salt Lake City, Utah, USA

${ }^{3}$ University of Utah, Salt Lake City, Utah, USA

\section{Correspondence to}

Dr Alli M Straubhar, Department of Obstetrics and Gynecology, The University of Utah, Salt Lake City, UT 84112, USA; alstraub@med.umich.edu

Received 9 April 2021 Accepted 28 July 2021 Published Online First 12 August 2021

\title{
Refusal of surgery and survival outcomes in endometrial cancer
}

\author{
Alli M Straubhar (D , ${ }^{1}$ Matthew W Parsons, ${ }^{2}$ Samual Francis, ${ }^{2}$ David Gaffney, ${ }^{2,3}$ Kathryn A Maurer ${ }^{1}$
}

\section{HIGHLIGHTS}

- Refusal of surgery in endometrial cancer is rare.

- Refusal of surgery has a negative impact on overall survival.

- In patients age $\leq 40$, refusal did not reach statistical significance to impact overall survival.

\section{ABSTRACT}

Objectives The goal of this study was to determine the impact refusal of surgery has on overall survival in patients with endometrial cancer.

Methods From January 2004 to December 2015, the National Cancer Database was queried for patients with pathologically proven endometrial cancer who were recommended surgery and refused. Inverse probability of treatment weighting was used to account for differences in baseline characteristics between patients who underwent surgery and those who refused. Kaplan-Meier analyses and doubly robust estimation with multivariate Cox proportional hazards modeling were used to analyze overall survival. Results of the 300675 patients identified, 534 patients $(0.2 \%)$ were recommended surgical treatment but refused: $18 \%$ (95/534) were age $\leq 40$ years. The 5 -year overall survival for all patients who refused surgery was significantly decreased compared with patients who underwent surgery $(29.2 \%$ vs $71.9 \%, \mathrm{P}<0.01)$. This was demonstrated at ages $41-64$ years $(65.5 \%$ vs $91.0 \%$, $\mathrm{P}<0.01)$ and $\geq 65$ years $(23.4 \%$ vs $75.3 \%, \mathrm{P}<0.01)$. The 5 year overall survival did not meet statistical significance at age $\leq 40$ years $(90.1 \%$ vs $87.8 \% \mathrm{P}<0.19)$. However, there were few patients in this cohort. On multivariate analysis, factors associated with refusal of surgery included: Medicaid insurance, Black race, Hispanic Race, Charlson Comorbidity Index scores of 2 or greater, stage II or III, and if patient received external beam radiation therapy alone. Factors associated with undergoing surgery included: age greater than 41 , stage IB, and if the patient received brachytherapy.

Conclusions Refusal of surgery for endometrial cancer is uncommon and leads to decreased overall survival.

\section{INTRODUCTION}

Of the estimated 66570 endometrial cancer cases diagnosed in 2021, the majority of the cases will occur in older patients with early-stage disease ${ }^{1}$ and over $80 \%$ of these patients will have a Type 1 malignancy. ${ }^{2}$ Young patients (less than age 44) account for approximately $9 \%$ of patients diagnosed annually. ${ }^{3}$ The estimated 5-year overall survival for endometrial cancer is $81 \%{ }^{4}$ The management of endometrial cancer is dictated by the suspected stage. For suspected uterine-confined disease, the mainstay of management is surgery with hysterectomy with bilateral salpingo-oophorectomy and lymphadenectomy if indicated. ${ }^{5}$

Patients may choose to not undergo surgery for a variety of reasons including patient preferences or physician bias $^{6}$ regarding treatment options. Young patients may refuse surgery seeking fertility-sparing options while older patients may be recommended an alternative therapy due to comorbidities or performance status. There is limited evidence in evaluating factors associated with refusal of surgery when recommended by the physician.

The objective of this study was to determine the impact of refusing recommended surgery on overall survival in patients with endometrial cancer using a large national database. Additionally, we sought to focus on the outcomes for younger and older patients and to describe what factors are associated with the refusal of surgery.

\section{METHODS}

The National Cancer Database, a joint project of the Commission on Cancer of the American College of Surgeons and the American Cancer Society, is a nationwide database on oncologic outcomes that collects information on $70 \%$ of all new invasive cancer diagnoses. ${ }^{7}$ The National Cancer Database and the hospitals participating in the National Cancer Database are the sources of the de-identified data used in this study. These data have not been verified. The National Cancer Database is not responsible for the validity or conclusions of this study. This database was selected because it offers a large, nationally applicable sample to examine with our query. Information within the database is pooled from a wide range of hospitals in diverse locations.

The National Cancer Database was used to identify patients aged 18 and older with pathologically proven and clinically or radiographically staged endometrial cancer who were recommended to undergo surgery yet refused from January 2004 to December 2015. The National Cancer Database defines whether a procedure has been performed or not and denotes 
reasons for not undergoing a surgical procedure, for which it has specific codes readily available. Of note, the National Cancer Database classifies dilation and curettage as an excisional biopsy and not a surgical procedure, therefore patients evaluated with this alone are not categorized as having undergone surgery. Patients who undergo a dilation and curettage and refuse hysterectomy would be considered to have refused surgery. ${ }^{8}$ Patients were excluded from the analysis if they had metastatic disease at time of diagnosis, had pre-invasive disease, such as complex atypical hyperplasia or endometrial intraepithelial neoplasia, non-epithelial histology (squamous cell carcinoma, melanoma, or sarcoma), or underwent neoadjuvant chemotherapy or radiation, International Federation of Gynecology and Obstetrics (FIGO) staging was missing or discordant, and if death or the date of the last contact occurred less than 3 months from diagnosis. Patients were also excluded if surgery was not performed for reasons other than patient refusal. These reasons included patients who were not medically fit for surgery, surgery not performed but reason is unclear, or if the patient passed away prior to surgery.

Covariates included age $(\leq 40,41-64, \geq 65)$, race and ethnicity (white, Black, Hispanic, other, unknown) insurance status (private insurance, Medicaid (state- provided healthcare coverage of eligible patients)), Medicare (a federal level insurance program for patients age $\geq 65$ or patients with disabilities), uninsured, unknown, community median household income quartile by zip code $(<\$ 38,000$, \$38000-47 999, \$48000-62 999, \$63 000+), hospital facility type, distance from residency zip code to hospital, urban density (urban, rural, unknown), and the percentile of residents within the zip code that had a high school degree. Comorbidities were estimated using the Charlson/Deyo composite comorbidity score $\left(0,1,2+\right.$, unknown). ${ }^{9-12}$ Tumors were classified by stage, grade, and histology. Alternative primary management strategies for the treatment of endometrial cancer were defined as receipt of chemotherapy or radiation therapy.

Differences in the distribution of demographic, clinical, and treatment characteristics among age groups were assessed using Pearson's chi-squared analysis. Propensity-score methods with inverse probability of treatment weighting was employed to account for imbalances in covariates between patients receiving and refusing surgery. ${ }^{1314}$ Characteristics matched by propensity-score included age, race, insurance status, income, urban density, education group, distance to care, Charlson-Deyo comorbidity score, grade, histology, FIGO substage, receipt of chemotherapy, and receipt of radiation. Univariate and multivariate logistic regression analyses were used to assess the predictive value for refusal of surgery. Factors with P-values $<0.05$ on univariate analysis were included in multivariate logistic regression models. Significance was defined as any $\mathrm{P}$-value $<0.05$.

The primary outcome variable was overall survival. Recurrence, progression-free survival, and toxicity were unable to be assessed as these data are not recorded within the National Cancer Database. The impact on overall survival was specifically evaluated by the following age categories: $\leq 40$ years, $41-64$ years, and $\geq 65$ years. Survival was assessed using Kaplan-Meier analyses and Cox proportional hazards modeling. A doubly-robust estimation was performed with multivariate Cox proportional hazards modeling on propensity-score matched cohorts to account for imperfect balancing of covariates. All matched variables were included within these doubly-robust multivariate models. All analyses were performed using the STATA 14.2 statistical package (Stata Corporation, College Station, TX).

\section{RESULTS}

\section{Demographics}

During the study period, the query of the National Cancer Database initially yielded 443680 patients. Patients were excluded if they had metastatic disease at diagnosis (427 880 remaining), had noninvasive disease, squamous cell carcinoma, melanoma, or sarcoma (383862 remaining), underwent neoadjuvant chemotherapy or radiation therapy (378841 remaining), FIG0 staging was missing or discordant (351754 remaining), surgery was not performed for reasons other than patient refusal (342334 remaining), and if death or the date of the last contact occurred less than 3 months from diagnosis (300675 remaining)). Of the 300675 patients identified during the study period, 534 patients $(0.2 \%)$ were recommended surgery but refused. Of the patients who refused surgery, $18 \%$ were aged $<41$ (95/534). Two-hundred and two patients (55\%) who refused surgery were clinically stage $1 \mathrm{~A}$. Patient and disease characteristics for the cohort are outlined in table 1.

\section{Characteristics Associated with Refusal of Surgery}

Table 2 demonstrates with univariate and multivariate analysis assessing the association of factors with refusal of surgery. On univariate analysis, factors associated with refusal of surgery include: no insurance, Medicaid, Medicare, Black race, Hispanic race, or categorized as Other race, Charlson Comorbidity Index scores of 2 or greater, stage II or III, and if the patient received external beam radiation therapy alone. Factors associated with less likelihood of refusing surgery included: age greater than 40 , income greater than $\$ 48000$, residing in an urban area, stage IB, moderately differentiated tumors, and if the patient received brachytherapy.

On multivariate analysis, factors associated with refusal of surgery included: Medicaid, Black race, Hispanic race, or categorized as Other race, Charlson Comorbidity Index scores of 2 or greater, stage II or III, and if the patient received external beam radiation therapy alone. Factors associated with undergoing surgery included: age greater than 41 , stage IB, and if the patient received brachytherapy.

\section{Survival Analysis}

For all patients who refused surgery, the 5 -year overall survival was significantly decreased $(29.2 \%$ vs $71.9 \%, P<0.01)$. When analyzing the cohort by age, a decreased overall survival was noted at ages 41-64 (65.5 vs $91.0 \%, \mathrm{P}<0.01)$ and for patients older than age $64(75.3 \%$ vs $23.4 \%, P<0.01)$. Five-year overall survival was not decreased in the younger cohort at less than age $41(90.1 \%$ vs $87.8 \%, \mathrm{P}<0.19$ ) (figure 1). Covariant-adjusted hazard ratios for death demonstrate only being age less than 41 was not associated with decreased survival (figure 2).

\section{DISCUSSION}

\section{Summary of Main Results}

According to a large national database, refusal of surgery for endometrial cancer is very rare. When patients refused surgery, they had lower overall survival. This decrease in survival was sustained in 


\section{Original research}

Table 1 Demographics of the cohort as categorized by patients who underwent surgery versus patients who refused surgery

\begin{tabular}{|c|c|c|}
\hline Characteristics & $\begin{array}{l}\text { Surgery } \\
n=300,141(98.8 \%)\end{array}$ & $\begin{array}{l}\text { Refused surgery } \\
n=534(0.2 \%)\end{array}$ \\
\hline \multicolumn{3}{|l|}{ Age } \\
\hline$\leq 40$ years & $10524(4)$ & $95(18)$ \\
\hline $41-64$ years & $169088(56)$ & $109(20)$ \\
\hline$\geq 65$ years & $120529(40)$ & $330(62)$ \\
\hline Race White & $246753(82)$ & $349(65)$ \\
\hline Black & $23624(8)$ & $108(20)$ \\
\hline Hispanic & $15321(5)$ & $42(8)$ \\
\hline Other & $10730(4)$ & $30(6)$ \\
\hline Unknown & $3713(1)$ & $5(1)$ \\
\hline Insurance status private & $154101(52)$ & $134(25)$ \\
\hline No insurance & $10305(3)$ & $29(5)$ \\
\hline Medicaid & $16577(6)$ & $55(10)$ \\
\hline Medicare & $114348(39)$ & $310(59)$ \\
\hline \multicolumn{3}{|l|}{ Income } \\
\hline$<\$ 38000$ & $46843(16)$ & $112(21)$ \\
\hline$\$ 38000-47,999$ & $68305(23)$ & $131(25)$ \\
\hline$\$ 48000-62,999$ & $81129(27)$ & $142(27)$ \\
\hline$\$ 63,000+$ & $101606(34)$ & $146(27)$ \\
\hline \multicolumn{3}{|l|}{ Distance from facility } \\
\hline Less than 50 miles & $253687(85)$ & $485(91)$ \\
\hline Greater than 50 miles & $44205(15)$ & $46(9)$ \\
\hline \multicolumn{3}{|l|}{ FIGO stage } \\
\hline IA & $170334(64)$ & $202(55)$ \\
\hline IB & $41154(15)$ & $23(6)$ \\
\hline II & $23501(9)$ & $66(18)$ \\
\hline IIIA & $9695(4)$ & $25(7)$ \\
\hline IIIB & $2435(1)$ & $38(10)$ \\
\hline IIIC & $18754(7)$ & $10(3)$ \\
\hline
\end{tabular}

patients older than 40 years of age but did not reach the threshold for statistical significance in younger patients.

\section{Results in the Context of Published Literature}

We surmise that a major reason why young patients would decline surgery is for fertility preservation. As more patients are delaying childbearing until the latter years of their reproductive life for social and cultural reasons, patients may face a diagnosis of endometrial cancer while being nulliparous. ${ }^{15}$ Due to this, investigations of fertility-sparing management strategies are becoming more paramount. Guidelines for fertility-sparing management of endometrial cancer recommend that the tumor be well differentiated and limited to the endometrium with the absence of metastatic disease including the absence of adnexal masses. ${ }^{16}{ }^{17}$ The patient also must be counseled that fertility-sparing treatments are not the standard of care. ${ }^{3}$

Progestin therapies are the mainstay for conservative hormonal management of patients desiring fertility-sparing management or in non-surgical candidates, and options include continuous progestin-based therapy oral options include medroxyprogesterone
Table 2 Univariant and multivariant relative risk (RR) for characteristics associated with refusal of surgery

\begin{tabular}{|c|c|c|}
\hline Characteristics $^{*}$ & $\begin{array}{l}\text { Univariant RR } \\
(95 \% \mathrm{Cl})\end{array}$ & $\begin{array}{l}\text { Multivariant RR } \\
(95 \% \mathrm{Cl})\end{array}$ \\
\hline \multicolumn{3}{|l|}{ Age } \\
\hline$\leq 40$ & 1 (reference) & 1 (reference) \\
\hline $41-64$ & 0.07 (0.05 to 0.09$)$ & $0.07(0.04$ to 0.1$)$ \\
\hline $65+$ & $0.3(0.2$ to 0.4$)$ & $0.27(0.17$ to 0.4$)$ \\
\hline Race White & 1 (reference) & 1 (reference) \\
\hline Black & 3.2 (2.6 to 4.0$)$ & 2.9 (2.1 to 4.1$)$ \\
\hline Hispanic & $1.9(1.4$ to 2.7$)$ & $2.0(1.2$ to 3.1$)$ \\
\hline Other & 2.0 (1.4 to 2.9 ) & 2.1 (1.2 to 3.6$)$ \\
\hline Insurance Insured & 1 (reference) & 1 (reference) \\
\hline No insurance & 3.2 (2.2 to 4.8$)$ & - \\
\hline Medicaid & 3.8 (2.8 to 5.2 ) & 2.0 (1.2 to 3.2$)$ \\
\hline Medicare & 3.1 (2.5 to 3.8 ) & - \\
\hline \multicolumn{3}{|l|}{ Income } \\
\hline$<\$ 38000$ & 1 (reference) & 1 (reference) \\
\hline$\$ 38000-47,999$ & - & - \\
\hline$\$ 48000-62,999$ & 0.7 (0.6 to 0.9$)$ & - \\
\hline$\$ 63,000+$ & 0.6 (0.5 to 0.8$)$ & - \\
\hline \multicolumn{3}{|l|}{ Urban density } \\
\hline Urban & 0.7 (0.5 to 1.0$)$ & - \\
\hline Rural & - & - \\
\hline \multicolumn{3}{|l|}{ Charlson Comorbidity Index } \\
\hline 1 & - & - \\
\hline $2+$ & 2.0 (1.4 to 2.8$)$ & 1.8 (1.1 to 2.9$)$ \\
\hline FIGO Stage IA & 1 (reference) & 1 (reference) \\
\hline IB & 0.5 (0.3 to 0.7$)$ & 0.4 (0.2 to 0.7$)$ \\
\hline ॥ & 2.4 (1.8 to 3.1$)$ & 1.9 (1.3 to 2.8$)$ \\
\hline III & 2.0 (1.5 to 2.6$)$ & 2.7 (1.8 to 4.0$)$ \\
\hline Grade well differentiated & 1 (reference) & 1 (reference) \\
\hline Moderate & 0.7 (0.6 to 0.9$)$ & - \\
\hline Poorly & - & - \\
\hline Undifferentiated & - & - \\
\hline \multicolumn{3}{|l|}{ Radiation therapy } \\
\hline $\begin{array}{l}\text { External beam alone } \\
\text { (EBRT) }\end{array}$ & 1.9 (1.5 to 2.4$)$ & 2.7 (1.9 to 4.0$)$ \\
\hline Brachytherapy & $0.3(0.2$ to 0.5$)$ & $0.5(0.2$ to 0.9$)$ \\
\hline
\end{tabular}

*Non-significant covariant levels not shown.

or megestrol acetate, ${ }^{18}$ or an intrauterine device containing levonorgestrel (LNG-IUD). ${ }^{19}{ }^{20}$ It is important to recognize the risk of the progression of disease and recurrence rates when considering fertility preservation. A recent meta-analysis investigated different efficacies of fertility-preservation strategies for achieving remission, recurrence rates, and pregnancy rates. Patients treated with a LNG-IUD followed by hormonal therapy had the lowest rates of recurrence $(11.0 \% ; 95 \% \mathrm{Cl} 5.1 \%$ to $22.0 \%)$ and highest rates of pregnancy $(56.0 \%, 95 \% \mathrm{Cl} 37.3 \%$ to $73.1 \%) .{ }^{21}$ In a recent publication that used the Surveillance, Epidemiology, and End Results (SEER) database demonstrated similar all-cause mortality and cancer-specific mortality in young patients age $<45$ years with 

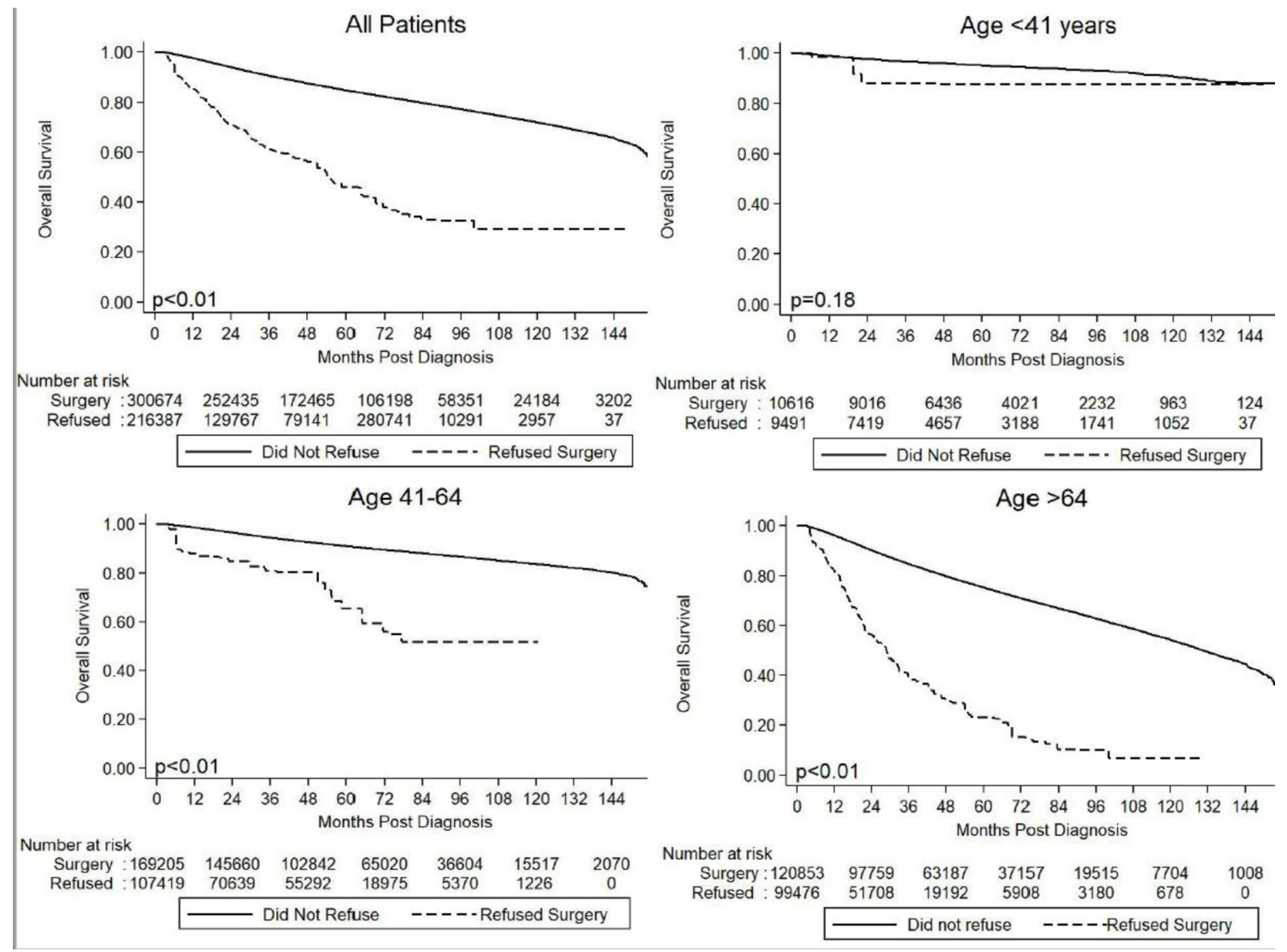

Figure 1 Kaplan-Meier curves for effect of refusal of surgery on overall survival for all patients and grouped by age.

localized, low-grade endometrial cancer treated with hormonal therapy compared with surgical management. ${ }^{22}$ In an observational study evaluating 148 patients aged less than 41 years of age with stage IA, grade 1, endometrial adenocarcinoma treated with oral progestins, the 5 -year survival for the cohort was $100 \%$ and the 5 -year recurrence-free survival was $68 \%(95 \% \mathrm{Cl} 58.5$ to 76.9$){ }^{23}$ The findings in our study, in addition to the aforementioned studies, suggest that in well selected (low-grade and low stage) and well counseled patients, fertility-sparing options are reasonable and do not have a lasting impact on overall survival. A registry for larger observational studies or randomized controlled trials are needed to delineate optimal management strategies for maximizing rates of remission and pregnancy outcomes.

When looking at variables associated with refusal of surgery, patients with stage II disease and greater were more likely to refuse surgery. Since these patients did not undergo surgical staging, this is based on clinical assessment. Clinical staging is a poor predictor of FIGO staging and discordance between stating and subsequent therapies can impact outcomes. ${ }^{24} \mathrm{~A}$ study by the Gynecologic Oncology Group (GOG) found only $24 \%$ of patients with clinical stage II disease had true surgical stage II disease.$^{25}$ Careful counseling of patients regarding potential staging discordance and its impact on outcomes is warranted.

In this study, minority populations were more likely to refuse surgery when compared with White patients (Black RR 2.9, (95\% Cl 2.1 to 4.1)), (Hispanic RR 2.0 (95\% Cl 1.2 to 3.1$)$ ). Similar findings were seen in a study evaluating racial disparities between White and Black patients with endometrial cancer using the SEER database. Black patients were less likely to undergo operative management when recommended $(79.8 \%$ vs $90.3 \%) .{ }^{26}$ This trend has been documented in refusal of surgery in other cancers including pancreatic and breast cancer. ${ }^{27}{ }^{28} \mathrm{~A}$ recent publication by Kaspers et $\mathrm{al}^{29}$ used the National Cancer Database to compare the quality of care in Black and White patients evaluating received and adherence to evidence-based treatment guidelines in endometrial cancer. This study showed that non-Hispanic Black patients and Hispanic patients were less likely to receive guideline-coordinated care compared with non-Hispanic White patients, and for the overall population, the lack of guideline coordinate care led to decreased overall survival. Other studies have shown that Black patients with endometrial cancer are less likely to receive the standard of care ${ }^{30}$ and more likely to die compared with their White counterpart. ${ }^{31}$ In 2014, a report was released by the Society of Gynecologic Oncology (SGO) Health Disparities Taskforce addressing disparities in gynecologic malignancies and provided potential strategies to overcome factors causing disparities such as providing outreach, awareness, and education of gynecologic malignancies. ${ }^{32}$ The results from this present study underscore the need for ongoing efforts to ensure these disparities continue to be addressed.

\section{Strenghts and Limitations}

Strengths of this project were the use of a large national database and the use of propensity score methods with inverse probability treatment weighting allowed for pairing of as many covariates as possible. Limitations of this study include its retrospective nature. Additionally, while 'patient refusal' is used to code the decision to refuse surgery, we cannot infer psychosocial or cultural factors that influence their decision or how the patient was counseled. While 


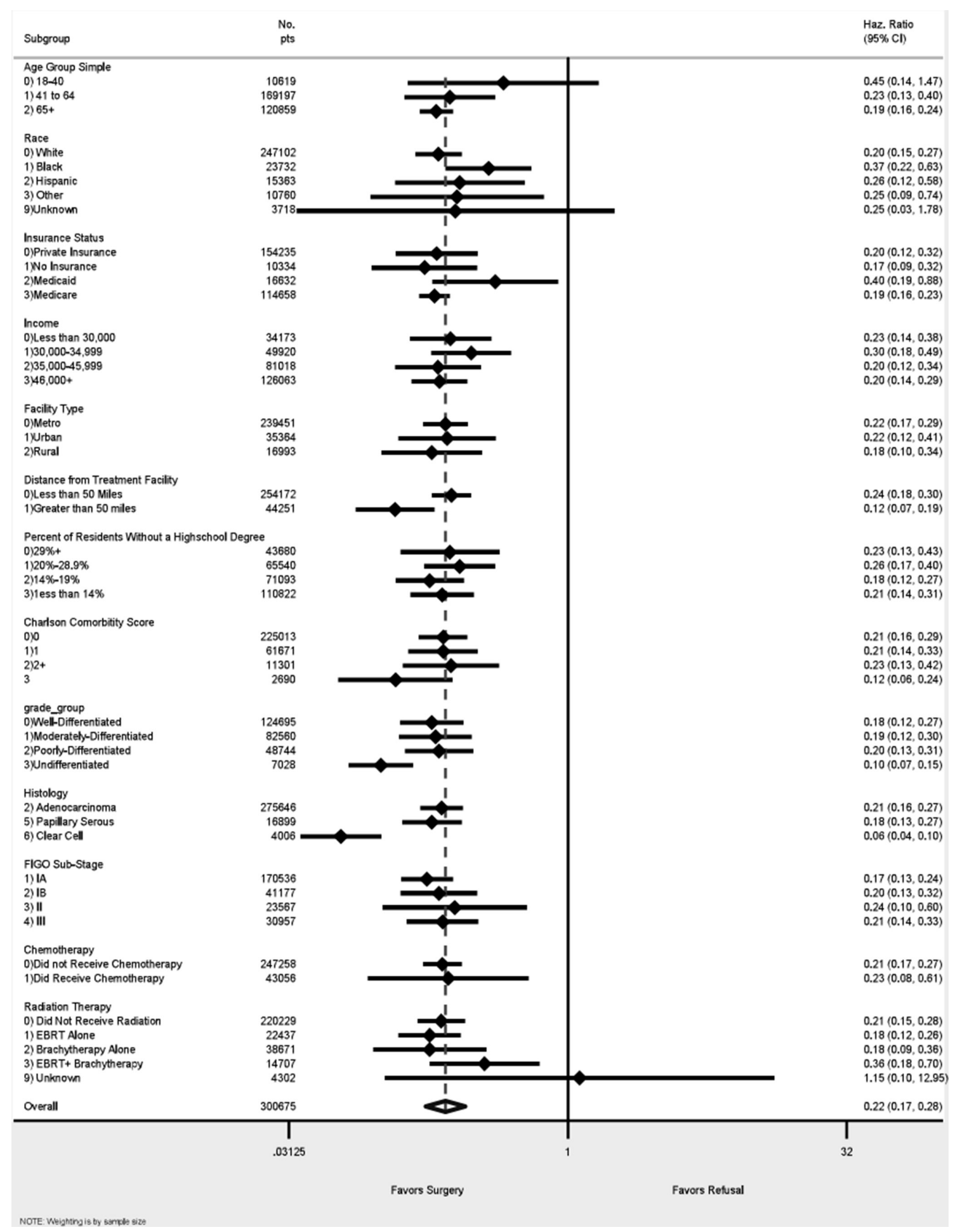

Figure 2 Adjusted hazard ratios for favor or refusal of surgery.

patients may be counseled and offered all modalities of treatment, we cannot comment on how patients are counseled and the impact this has on their decision for refusal of this treatment. Furthermore, we are limited in our knowledge of how the young patients who refused surgery were treated. There is also potential bias in who is coded as refusing surgery, where if shared decision-making between patient and provider led to treatment with hormonal therapy, this patient may not be coded as refusing surgery. If a physician feels strongly that surgery is the recommended choice and the patient turns down that recommendation in favor of hormonal therapy, that would likely be considered refusal of surgery.

\section{Implications for Practice and Future Research}

It is important to consider the small number of patients that refused surgery in our study and in other studies reviewed above. To date, there is no level I evidence available regarding the impact refusal of surgery or medical management with hormonal therapy have on overall survival in young patients with endometrial cancer. Due to 
the rarity of these cases, potentially a national registry or database can help accumulate more patients for observational data. Ideally, prospective randomized clinical trials are needed in this specific age group to determine primary treatment strategies' impact on overall survival and to determine which methods of hormonal therapy led to the desired outcome of fertility.

\section{CONCLUSIONS}

In summary, using a national database illustrated that refusal of surgery for endometrial cancer is rare. Overall, refusal of surgery resulted in worse outcomes for the entire cohort, particularly in the older patient cohorts. In young patients in this cohort, refusal of surgery did not reach statistical significance to show an impact overall survival.

Acknowledgements Outside the submitted work, Dr. Straubhar has a patent W02019195097A1 perineal heating device issued.

Contributors Conception and design: AMS, MWP, KAM, DG. Data analysis and interpretation: AMS, MWP, SF, KAM. Manuscript writing: AS, MP, KAM. Final approval of manuscript: AMS, MWP, KAM, DG, SF.

Funding The authors have not declared a specific grant for this research from any funding agency in the public, commercial, or not-for-profit sectors.

Competing interests None declared.

Patient consent for publication Not required.

Provenance and peer review Not commissioned; externally peer reviewed.

Data availability statement Data are available upon reasonable request. Data were obtained from the National Cancer Database.

ORCID iD

Alli M Straubhar http://orcid.org/0000-0001-6657-5016

\section{REFERENCES}

1 Siegel RL, Miller KD, Jemal A. Cancer statistics, 2019. CA Cancer J Clin 2019;69:7-34.

2 Garg K, Soslow RA. Endometrial carcinoma in women aged 40 years and younger. Arch Pathol Lab Med 2014;138:335-42.

3 SGO Clinical Practice Endometrial Cancer Working Group, Burke WM, Orr J, et al. Endometrial cancer: a review and current management strategies: part II. Gynecol Oncol 2014;134:393-402.

4 Seer Cancer Stat Facts: endometrial cancer, 2019. Available: http:// seer.cancer.gov/statfacts/html/corp.html [Accessed January 7 2020]

5 SGO Clinical Practice Endometrial Cancer Working Group, Burke WM, Orr J, et al. Endometrial cancer: a review and current management strategies: part I. Gynecol Oncol 2014;134:385-92.

6 Eggemann $\mathrm{H}$, Ignatov $\mathrm{T}$, Burger $\mathrm{E}$, et al. Management of elderly women with endometrial cancer. Gynecol Oncol 2017;146:519-24.

7 Bilimoria KY, Stewart AK, Winchester DP, et al. The National Cancer Database: a powerful initiative to improve cancer care in the United States. Ann Surg Oncol 2008;15:683-90.

8 National Cancer Database participant user file (PUF) data dictionary. version: PUF 2016 - containing cases diagnosed in 2004-2016, 2016. Available: https://www.facs.org/quality-programs/cancer/ ncdb/puf

9 Charlson ME, Pompei P, Ales KL, et al. A new method of classifying prognostic comorbidity in longitudinal studies: development and validation. J Chronic Dis 1987;40:373-83.

10 Deyo RA, Cherkin DC, Ciol MA. Adapting a clinical comorbidity index for use with ICD-9-CM administrative databases. J Clin Epidemiol 1992;45:613-9.
11 Medicaid. Available: https://www.medicaid.gov/medicaid/index.html [Accessed 20 July 2021].

12 What's Medicare? Available: https://www.medicare.gov/whatmedicare-covers/your-medicare-coverage-choices/whats-medicare [Accessed 20 July 2021].

13 Hemmila MR, Birkmeyer NJ, Arbabi S, et al. Introduction to propensity scores: a case study on the comparative effectiveness of laparoscopic vs open appendectomy. Arch Surg 2010;145:939-45.

14 Ruiz MP, Huang Y, Hou JY, et al. All-cause mortality in young women with endometrial cancer receiving progesterone therapy. Am J Obstet Gynecol 2017;217:669.e1-669.e13.

15 Rodolakis A, Biliatis I, Morice P, et al. European Society of Gynecological Oncology Task Force for Fertility Preservation: clinical recommendations for fertility-sparing management in young endometrial cancer patients. Int J Gynecol Cancer 2015;25:1258-65.

16 Zivanovic O, Carter J, Kauff ND, et al. A review of the challenges faced in the conservative treatment of young women with endometrial carcinoma and risk of ovarian cancer. Gynecol Oncol 2009;115:504-9.

17 Eskander RN, Randall LM, Berman ML, et al. Fertility preserving options in patients with gynecologic malignancies. Am J Obstet Gynecol 2011;205:103-10.

18 Ramirez PT, Frumovitz M, Bodurka DC, et al. Hormonal therapy for the management of grade 1 endometrial adenocarcinoma: a literature review. Gynecol Oncol 2004;95:133-8.

19 Gunderson CC, Fader AN, Carson KA, et al. Oncologic and reproductive outcomes with progestin therapy in women with endometrial hyperplasia and grade 1 adenocarcinoma: a systematic review. Gynecol Oncol 2012;125:477-82.

20 Hubbs JL, Saig RM, Abaid LN, et al. Systemic and local hormone therapy for endometrial hyperplasia and early adenocarcinoma. Obstet Gynecol 2013;121:1172-80.

21 Fan Z, Li H, Hu R, et al. Fertility-preserving treatment in young women with grade 1 presumed stage IA endometrial adenocarcinoma: a meta-analysis. Int J Gynecol Cancer 2018;28:385-93.

22 Greenwald ZR, Huang LN, Wissing MD, et al. Does hormonal therapy for fertility preservation affect the survival of young women with early-stage endometrial cancer? Cancer 2017;123:1545-54.

23 Park J-Y, Kim D-Y, Kim J-H, et al. Long-term oncologic outcomes after fertility-sparing management using oral progestin for young women with endometrial cancer (KGOG 2002). Eur J Cancer 2013;49:868-74.

24 van Lankveld MAL, Koot NCM, Peeters PHM, et al. Compliance to surgical and radiation treatment guidelines in relation to patient outcome in early stage endometrial cancer. J Eval Clin Pract 2006;12:196-201.

25 Creasman WT, DeGeest K, DiSaia PJ, et al. Significance of true surgical pathologic staging: a Gynecologic Oncology Group study. Am J Obstet Gynecol 1999;181:31-4.

26 Sud S, Holmes J, Eblan M, et al. Clinical characteristics associated with racial disparities in endometrial cancer outcomes: a surveillance, epidemiology and end results analysis. Gynecol Oncol 2018;148:349-56.

27 Tohme S, Kaltenmeier C, Bou-Samra P, et al. Race and health disparities in patient refusal of surgery for early-stage pancreatic cancer: an NCDB cohort study. Ann Surg Oncol 2018;25:3427-35.

28 Gaitanidis A, Alevizakos M, Tsalikidis C, et al. Refusal of cancerdirected surgery by breast cancer patients: risk factors and survival outcomes. Clin Breast Cancer 2018;18:e469-76.

29 Kaspers M, Llamocca E, Quick A, et al. Black and hispanic women are less likely than white women to receive guideline-concordant endometrial cancer treatment. Am J Obstet Gynecol 2020;223:398. e1-398.e18.

30 Rauh-Hain JA, Melamed A, Schaps D, et al. Racial and ethnic disparities over time in the treatment and mortality of women with gynecological malignancies. Gynecol Oncol 2018;149:4-11.

31 Long B, Liu FW, Bristow RE. Disparities in uterine cancer epidemiology, treatment, and survival among African Americans in the United States. Gynecol Oncol 2013;130:652-9.

32 Collins Y, Holcomb K, Chapman-Davis E, et al. Gynecologic cancer disparities: a report from the Health Disparities Taskforce of the Society of Gynecologic Oncology. Gynecol Oncol 2014;133:353-61. 